\title{
Pathological Comparisons of the Hippocampal Changes in the Transient and Permanent Middle Cerebral Artery Occlusion Rat Models
}

\begin{abstract}
Fawad Ali Shah 1,2†, Tao Li ${ }^{3+}$, Lina Tariq Al Kury ${ }^{4}$, Alam Zeb ${ }^{2}$, Shehla Khatoon, Gongping Liu ${ }^{6,7}$, Xifei Yang ${ }^{8}$, Fang Liu ${ }^{9,10}$, Huo Yao ${ }^{1}$, Arif-Ullah Khan ${ }^{2}$, Phil Ok Koh ${ }^{11}$, Yuhua Jiang ${ }^{12 *}$ and Shupeng $L^{1,9,10 *}$

1 State Key Laboratory of Oncogenomics, School of Chemical Biology and Biotechnology, Peking University Shenzhen Graduate School, Shenzhen, China, ${ }^{2}$ Department of Pharmacology, Riphah Institute of Pharmaceutical Sciences, International University, Islamabad, Pakistan, ${ }^{3}$ Department of Forensic Medicine, School of Medicine, Xi'an Jiaotong University, Xi'an, China, ${ }^{4}$ College of Natural and Health Sciences, Zayed University Abu Dhabi, Abu Dhabi, United Arab Emirates, ${ }^{5}$ Department of Anatomy, Khyber Medical College, Khyber Medical University, Peshawar, Pakistan, ${ }^{6}$ Department of Pathophysiology, School of Basic Medicine and the Collaborative Innovation Center for Brain Science, Key Laboratory of Ministry of Education of China and Hubei Province for Neurological Disorders, Tongji Medical College, Huazhong University of Science and Technology, Wuhan, China, ${ }^{7}$ Co-innovation Center of Neuroregeneration, Nantong University, Nantong, China, ${ }^{8}$ Key Laboratory of Modern Toxicology of Shenzhen, Shenzhen Center for Disease Control and Prevention, Shenzhen, China, ${ }^{9}$ Department of Psychiatry, University of Toronto, Toronto, ON, Canada, ${ }^{10}$ Centre for Addiction and Mental Health, Campbell Research Institute, Toronto, ON, Canada, ${ }^{11}$ Department of Anatomy, College of Veterinary Medicine, Research Institute of Life Science, Gyeongsang National University, Jinju-si, South Korea, ${ }^{12}$ Cancer Centre, The Second Hospital of Shandong University, Jinan, China
\end{abstract}

Ischemic strokes are categorized by permanent or transient obstruction of blood flow, which impedes delivery of oxygen and essential nutrients to brain. In the last decade, the therapeutic window for tPA has increased from 3 to $5-6 \mathrm{~h}$, and a new technique, involving the mechanical removal of the clot (endovascular thrombectomy) to allow reperfusion of the injured area, is being used more often. This last therapeutic approach can be done until $24 \mathrm{~h}$ after stroke onset. Due to this fact, more acute ischemic stroke patients are now being recanalized, and so tMCAO is probably the "best" model to address these patients that have a potential good outcome in terms of survival and functional recovery. However, permanent occlusion patients are also important, not only to increase survival rate but also to improve functional outcomes, although these are more difficult to achieve. So, both models are important, and which target different stroke patients in the clinical scenario. Hippocampus has a vital role in memory and cognition, is prone to ischemic induced neurodegeneration. This study was designed to delineate the molecular, pathological, and neurological changes in rat models of t-MCAO, permanent MCAO (pMCAO), and PMCAO with diabetic conditions in hippocampal tissue. Our results showed that these three models showed distinct discrepancies at numerous pathological process, including key signaling molecules involved in neuronal apoptosis, glutamate induced excitotoxicity, neuroinflammation, oxidative stress, and neurotrophic changes. Our result suggests that the two commonly used MCAO models exhibited tremendous differences in terms of neuronal cell loss, glutamate excitotoxic 
related signaling, synaptic transmission markers, neuron inflammatory and oxidative stress molecules. These differences may reflect the variations in different models, which may provide valuable information for mechanistic and therapeutic inconsistences as experienced in both preclinical models and clinical trials.

Keywords: ischemic stroke, hippocampus, diabetes, transient and permanent cerebral ischemia, neurodegeneration, glutamate receptor, inflammation, ROS

\section{INTRODUCTION}

Stroke accounts for great number of death and disability across the globe. The frequency of stroke varies with demographic location, and generally considered to be the 2nd leading cause of mortality in industrialized countries. Ischemic stroke represents the frequently encounter stroke type, caused by thrombosis or dislodged emboli. The characteristic events in ischemic stroke include extensive depolarization, release of excitatory glutamate, opening of voltage gated ion channels, and intracellular $\mathrm{Ca}^{2+}$ buildup. Induced by intraluminal suture, focal cerebral ischemia is a well-established animal stroke model of clinical relevance, comprising of transient ( $\mathrm{t}-\mathrm{MCAO}$ ) and permanent ( $\mathrm{p}-\mathrm{MCAO}$ ). $\mathrm{t}$ MCAO is extensively employed in about $88 \%$ basic experimental MCAO models from 2014 to 2015 (1). Despite of several advantages (2-4), many consistent studies suggested some shortfall to t-MCAO $(5,6)$. Contrary, p-MCAO mimics the large vessel occlusion (LVO) due to no reperfusion process, and is equally important. However, permanent occlusion is more difficult to achieve and it is associated with increase mortality attributed to higher swelling and intracranial pressure. So, both models are important, and target different stroke patients in the clinical scenarios.

Pathological changes of both t-MCAO and p-MCAO models included core region where blood flow dropped from 10 to $25 \%$ and is composed of neuronal necrosis, surrounded by penumbra region where brain tissue suffered from mild to moderate ischemic damage. The infarcted region in rat comprised primarily of cortex, striatum, thalamus and hypothalamus, while in mice the infarction also extent to hippocampus $(7,8)$. Moreover, sensitivity of different brain regions to ischemia varies based on collateral circulation, rodent strain, and experimental set up such as t-MCAO and p-MCAO (9-11). Many studies confined ischemic core to striatum when MCA occluded for $30 \mathrm{~min}$, increasing ischemic interval will proportionally expends core and penumbral boundaries $(12,13)$. As a result, t-MCAO

\footnotetext{
Abbreviations: MCAO, middle cerebral artery occlusion; I/R, ischemic reperfusion; Casp-3, caspase 3; HSP70, heat shock protein; BDNF, brain derived neurotrophic factor; NMDAR, N-methyl-d-aspartate receptor; TTC, 2,3,5-triphenyltetrazolium chloride; BCA, bicinchoninic acid; PVDF, polyvinylidene fluoride; TBST, tris-buffered saline containing $0.1 \%$ Tween-20; CST, cell signaling technology; DAB, 3, 3'-diaminobenzidine tetra hydrochloride; SNAP-25, synaptosomal associated protein 25 ; GSK-3 $\beta$, glycogen synthase kinase$3 \beta$; Glu, glutamate; BDP, break down products; PSD95, post-synaptic density protein; NR2a, N-methyl D-aspartate 2A; NR2b, N-methyl D-aspartate 2b; ERK, extracellular-signal-regulated kinase; FJB, Fluoro-Jade B; Synapto, synaptophysin; $\mathrm{NF}_{\mathrm{K}} \mathrm{B}$, nuclear factor kappa-light-chain-enhancer of activated $\mathrm{B}$ cells; $\mathrm{TNF} \alpha$, tumor necrosis factor alpha; COX2, cyclooxygenase; ROS, reactive oxygen species; GFAP, glia fibrillary acid binding protein; VEGF, vascular endothelial growth factor.
}

and p-MCAO models represented different advantages coherent to both the clinical scenario and underlying mechanisms.

There are limited studies on hippocampus as per our information and literature study using permanent MCAO (p$\mathrm{MCAO}$ ) approach. Few studies demonstrated morphological and biochemical variations in hippocampus following p-MCAO (1416) The hippocampus in rodents is supplied by anterior choroidal artery (a branch form ICA) and posterior hippocampal artery (branch arising from posterior communication artery, PcomA) (17). As such, the intraluminal blockage will hinder blood supply in choroidal artery, with minimum effect on flow in posterior hippocampal artery. Moreover, various factors influencing the extent of ischemic stroke, such as suture/nylon filament diameter (3-0, 4-0), length of filament, coating by (silicone or poly-Llysine), and tip of filament (18-20). Among, duration of artery occlusion has a more pronounced effect on volume of infarction $(12,13)$.

In the present study, we have compared the molecular changes in rat models of t-MCAO, p-MCAO, and pMCAO with diabetic condition in hippocampus. Diabetes is an independent risk factor and important comorbid of stroke, which not only increased the risk of ischemic stroke to 1.5-3 times, but also worsened the stroke outcomes with increased aggravated neurological deficits, functional disabilities, and mortality $(21,22)$. Our objective is to determined hippocampus response after transient and permanent ischemia and secondly to delineate inconsistencies in neuronal apoptosis, excitotoxicity, neuroinflammation, and neurogenesis in rat models of t-MCAO, p-MCAO, and p-MCAO with diabetic condition as these discrepancies may partially explain repetitive failures of experimental findings during clinical trials. Thus, further characterizing the detailed molecular and cellular processes will unveil the complex pathological processes and will provide the basis for more coherent clinical interventions.

\section{EXPERIMENTAL PROCEDURES}

\section{Animals Grouping and Drug Treatment}

Adult male Sprague-Dawley rats weighing 200-230 g (8-9 weeks) were purchased from Guangdong Medical Laboratory Animal Center, China. The experimental animals were housed at Laboratory Animal Research Center, Peking University Shenzhen Graduate School, under $12 \mathrm{~h}$ light $/ 12 \mathrm{~h}$ dark cycle at $18-22^{\circ} \mathrm{C}$ and had free access to diet and tap water throughout the study. The experimental procedures were set in such a way to minimize rats suffering. All experimental procedures were carried out according to the protocols approved by Institutional Animal Care 
and Use Committee of Peking University Shenzhen Graduate School. We did not use any blind allocation or randomization of rats, instead we adhered the criteria to keep similar weight animal to same group under the same experimental condition. The rats were randomly divided into 4 groups ( $n=15$ /group) containing: Sham operated control group; transient middle cerebral artery occlusion for $90 \mathrm{~min}$ followed by reperfusion group (I/R$\mathrm{MCAO}$ ); permanent middle cerebral artery occlusion group (p$\mathrm{MCAO);} \mathrm{permanent} \mathrm{middle} \mathrm{cerebral} \mathrm{artery} \mathrm{occlusion} \mathrm{in} \mathrm{diabetic}$ rat group (Dia p-MCAO). Streptozotocin (40 mg/kg, Sigma, St. Louis, MO, U.S.A.) dissolved in citrate buffer $(0.1 \mathrm{mM}, \mathrm{pH} 4.2)$ and injected intraperitoneally to induce diabetic symptoms (23). Blood glucose levels were determined using (Accu-Chek-Roche Diagnostics, Mannheim, Germany) and diabetes was defined as fasting blood glucose $>300 \mathrm{mg} / \mathrm{dL}$.

\section{MCAO Surgery}

MCAO procedure were operated as previously described (2426). Briefly, rats were anesthetized by mixture of xylazine and ketamine $(1: 3.2, \mathrm{I} / \mathrm{P})$. The body temperatures of rats were maintained at $37 \pm 1^{\circ} \mathrm{C}$ by using blanket and heating lamps. Briefly, a cervical incision was achieved on ventral side, keeping the incision laterally toward right region. The underlying tissues were carefully dissected to locate the right common carotid artery (CCA), and which was further set free from thin vagus nerve run laterally to CCA. The two bifurcating branches of CCA, external and internal carotid artery were identified and set free from surrounding tissues. The thin smaller arteries, occipital artery, and superior thyroid artery arising from external carotid artery were ligated with black silk (6/0) and subsequently pierced. A permanent knot was applied to external carotid artery above the origin of superior thyroid artery near hyoid bone. Moreover, the external carotid artery was cut by a sharp scissor near the bifurcating point, and immediately a thick nylon silk with dimension (3/0) having length $3 \mathrm{~cm}$ while keeping the tip of the silk round manually by heat, was inserted from the opening of external carotid artery and advanced further into internal carotid artery to the origin of middle cerebral artery (MCA), whereas a small resistance indicated the occlusion of MCA. Twentyfour hours after occlusion, all animals were killed for sample collection. The sham group was exposed to similar measures but with no nylon insertion. The filament remained in placed in rats undergoing $\mathrm{p}-\mathrm{MCAO}$ but removed $90 \mathrm{~min}$ later after stroke onset in transient ischemic rats (Figure 1A). All ischemic rats were returned to the cages and were observed for $24 \mathrm{~h}$. Ten rats were died during the experiment including 3 from $\mathrm{p}$ $\mathrm{MCAO}$ group, 2 from $\mathrm{t}-\mathrm{MCAO}, 5$ from Dia $+\mathrm{p}-\mathrm{MCAO}$, which we excluded from the study. Various methodologies are being used for stroke induction, broadly classified into craniotomies methods using photothrombosis and electrocoagulation. Both kind of transient and permanent occlusion can be induced by this. A major disadvantage associated with these procedures are large craniotomies and sometimes damage to skin and skull structures. Moreover, the intraluminal method using blue nylon silk is widely used in experimental procedures, though the major limitation associated with this model is subarachnoid hemorrhage due to vessel rupturing and hyperthermia.

\section{TTC Staining}

TTC staining was performed as previously described (27). Briefly, brain tissues were carefully removed and washed with cold PBS. Three to four millimeter-thick coronary sections were cut by using sharp blade from frontal lobe. These coronal slices were incubated in 2\% TTC for 10-20 min, until a thorough demarcation was observed for MCAO operated rats; while sham operated rats were stained deep red. ImageJ software was used to quantitatively determine infarcted area by optimizing background of image to threshold intensity and then calculated infarct area from total brain area. To compensate for brain edema, the corrected brain infarction was calculated as follow:Corrected infarct area $=$ left hemisphere area - (right hemisphere area - infarct area).

\section{Western Blot}

For Western blot analysis, the samples were dissolved in lysis buffer ( $1 \mathrm{M}$ Tris-HCI, $5 \mathrm{M}$ sodium chloride, $0.5 \%$ sodium deoxycholate, $10 \%$ sodium dodecyl sulfate, $1 \%$ sodium azide, $10 \%$ NP-40) (28). The homogenate was sonicated, centrifuged and protein concentration was determined by BioRad protein assay kit (BioRad Laboratories, CA, and USA) according to guidelines provided by manufacturer. Equal amount of protein (i.e., $30 \mu \mathrm{g}$ per sample) were electrophoresed on $10 \%$ SDS-PAGE gels followed by immunoblotting for transferring the protein to poly-vinylidene fluoride (PVDF) membranes (Millipore, Billerica, MA, USA). PVDF was washed in Tris-buffered saline containing $0.1 \%$ Tween-20 (TBST) and then incubated with primary antibodies overnight at $4{ }^{\circ} \mathrm{C}$. The membranes were then incubated with appropriate secondary antibody, and protein band were detected using an ECL detection reagent according to the manufacturer's instructions (Amersham Pharmecia Biotech, Uppsala, Sweden). The X-ray films were scanned, and optical densities of the bands were analyzed through densitometry using computer-based ImageJ program.

The antibodies used include anti-HSP70 (SC-66048), antiBcl2 (SC-2960), anti-Caspase3 (SC-7148), anti-NRF2 (SC-722), anti- HO-1 (SC-136961), anti-p-JNK (SC-6254), anti-JNK (SC7345), anti-P38 (SC-7972), anti-ERK (SC-135900), anti-NR2a (SC-1468), anti-NR2b (SC-1469), anti-PSD95 (SC-71933), antiGluR1 (SC-55509), anti-p-GluR1 serine831 (SC-16313), anti SNAP25 (SC-7538), anti-Synaptophysin (SC-17750), anti-BDNF (SC-546), anti-VEGF (SC-7269), anti-p-NF $\mathrm{N}_{\mathrm{k}} \mathrm{B}$, anti-TNF (SC52746), anti-GFAP (SC-33673), anti-COX2 (SC-7951), and anti$\beta$-Actin from (Santa Cruz, Biotechnology, CA, USA) at dilution of 1:1,000. Anti-p-P38 (Cat \# 4511), anti-p-ERK (Cat \# 8544), and anti-p-GluR1serine845 (Cat \# 8084) were purchased from Cell Signaling.

\section{Immunohistochemistry}

Brain tissues were fixed in $4 \%$ paraformaldehyde and embedded in paraffin, and $4 \mu \mathrm{m}$ coronary sections were cut using a rotary microtome (29). Tissue sections on coated slides were de-paraffinized, with three different absolute xylenes and were rehydrated with ethyl alcohol [from 100\% (absolute) to $70 \%$ ]. The slides were rinsed with distilled water and immersed in $0.01 \mathrm{M}$ phosphate-buffered saline (PBS) for $10 \mathrm{~min}$. The slides 
A

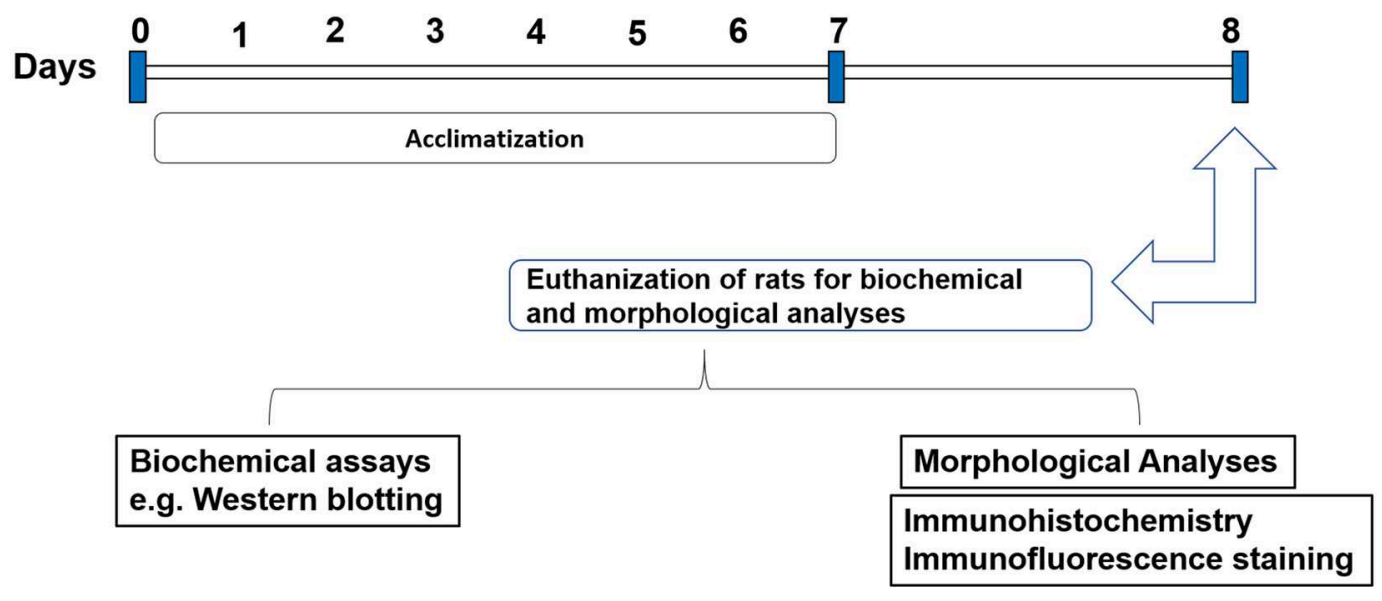

Rats grouping and therapeutic approach

i) Sham group

ii) I/R MCAO group

iii) p-MCAO group

iv) Dia p-MCAO group
Euthanization of rats for biochemical and morphological analyses
B
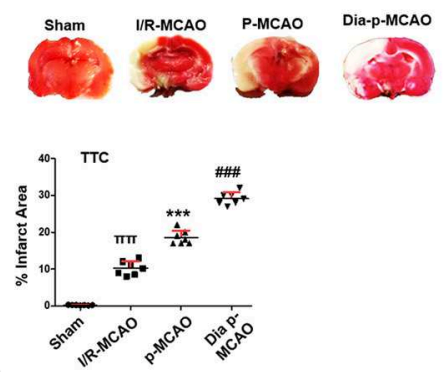

D

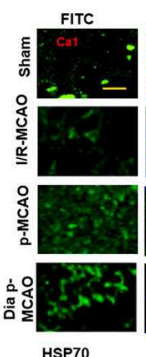

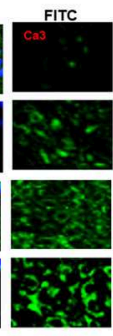

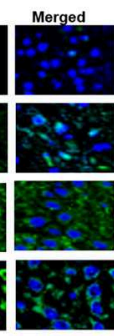

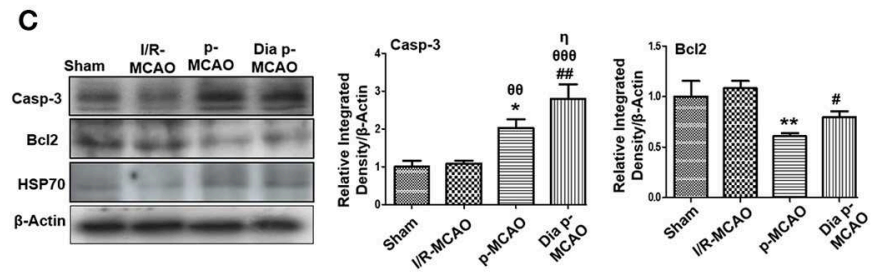

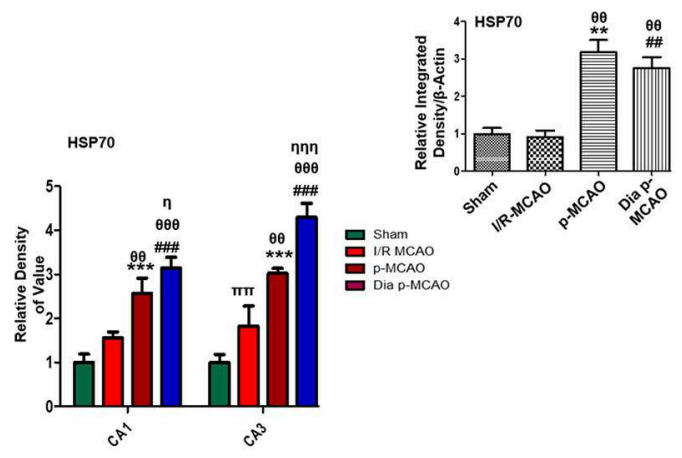

FIGURE 1 | Relative effect of ischemia on brain infarction and cell apoptosis (A) Flow chart of experiment. (B) Brain coronal sections were stained with TTC, which distinguishes between ischemic and non-ischemic areas, one ways ANOVA followed by post-hoc bonferroni multiple comparison test using graph-pad prism- 5 software ( $n=7 /$ group). ${ }^{*} p<0.05$, ${ }^{\#} p<0.05$, and ${ }^{\pi} p<0.05$ relative to sham group. (C) Western blot analysis of Casp-3, Bcl2, HSP70. Densitometric analysis was expressed in relative to $\beta$-Actin $\left(n=5\right.$ /group). ${ }^{*} p<0.05$ and ${ }^{\#} p<0.05$ relative to sham group, ${ }^{\theta} p<0.05$ relative to I/R MCAO, ${ }^{n} p<0.05$ between $p$-MCAO and Dia $\mathrm{p}-\mathrm{MCAO} .{ }^{\theta \theta}$ shows significant difference of $\mathrm{p}-\mathrm{MCAO}$ and Dia $\mathrm{p}-\mathrm{MCAO}$ to $\mathrm{I} / \mathrm{R}$, and its value is $p<0.01,{ }^{\theta \theta \theta}$ shows significant difference of $\mathrm{p}-\mathrm{MCAO}$ and Dia $\mathrm{p}-\mathrm{MCAO}$ to $\mathrm{I} / \mathrm{R}$, and its value is $p<0.001$, ${ }^{\pi \pi}$ shows significant difference of $\mathrm{I} / \mathrm{R} \mathrm{MCAO}$ to sham, and its value is $p<0.01$, ${ }^{\eta \eta \eta}$ shows significant difference between $p-M C A O$ and Dia p-MCAO, and its value is $p<0.001$. (D) Representative images of HSP70 immunofluorescence staining in CA1 and CA3 region of hippocampus ( $n=5 /$ group). Scale bar $=100 \mu \mathrm{m}$. ${ }^{*} p<0.05$ and ${ }^{\#} p<0.05$ relative to sham group, ${ }^{\theta} p<0.05$ relative to I/R MCAO, ${ }^{n} p<0.05$ between $p-M C A O$ and Dia $p-M C A O .{ }^{* *} p<0.01$, ${ }^{* \star *} p<0.001, \# \# p<0.01$, and $\# \# \# p<0.001$. 
were processed for antigen retrieval step using a heat method. The slides were allowed to cool and washed with PBS twice times. After antigen retrieval, the slides were incubated with $3 \%$ hydrogen peroxidase to quench endogenous peroxidase and were subsequently blocked with $5 \%$ serum depending upon the sources of secondary antibodies used. After blocking, the slides were incubated overnight in mouse anti-VEGF (SC-7269, Santa Cruz Biotechnology) at 1:100 dilution. followed by treatment with appropriate biotinylated secondary antibodies for $2 \mathrm{~h}$ and successively with ABC reagents (Santa Cruz Biotechnology) for $1 \mathrm{~h}$ at room temperature. The sections were washed with PBS and stained in 3, 3'-Diaminobenzidine tetrahydrochloride solution; they were then washed with distilled water, dehydrated in graded ethanol (70, 95, and 100\%), fixed in xylene, and cover-slipped by a mounting medium. Immunohistochemical results were analyzed by a light microscope (Olympus, Japan), which was connected to a digital photomicroscopy system. ImageJ software was used to quantitatively determine hyperactivated VEGF, by optimizing background of image to threshold intensity and analyzing cytoplasmic VEGF positive cells at the same threshold intensity for all groups and was expressed as the relative integrated density of the samples relative to the sham.

\section{Fluoro-Jade B Staining}

The slides were immersed in a solution of $1 \%$ sodium hydroxide, graded ethanol, and then in distilled water. Slides were transferred into a coplin jar and were washed with $0.06 \%$ potassium permanganate solution for $10 \mathrm{~min}$. The slides were rinsed with distilled water, and then transferred to a $0.01 \%$ Fluoro-Jade B solution containing $0.1 \%$ acetic acid. The slides were then washed with distilled water and air dried. The slides were incubated with DAPI and cover slipped with nonfluorescent mounting media and photographed. ImageJ software was used to quantitatively determine fluorescence intensity of hippocampus/total area for all groups. The immunofluorescence intensity was expressed as the relative integrated density of the samples relative to the sham.

\section{Immunofluorescence Analysis}

After de-paraffinization of sections, the slides were autoclaved in $0.1 \mathrm{M}$ sodium citrate $\mathrm{pH} 6$ for antigen retrieval step (30). The slides were allowed to cool and washed with PBS twice times. Slides were incubated with $5 \%$ normal serum depending upon the source of secondary antibody used. The slides were incubated with primary antibodies at $4{ }^{\circ} \mathrm{C}$ overnight (HSP70, 8-oxoguanine p-JNK, COX2, GFAP) from Santa Cruz Biotechnology at 1:100 dilution. Next morning, after washing with PBS, fluorescent labeled secondary antibodies (Santa Cruz Biotechnology) as 1:50 dilution were used for signal amplification in dark chamber, followed by mounted with UltraCruz mounting medium (Santa Cruz Biotechnology). The slides were pictured with confocal scanning microscopes (Flouview FV 1000, Olympus, Japan) and fluorescence intensity was quantitatively analyzed by ImageJ and expressed as the relative integrated density of the samples relative to the sham.

\section{Statistical Analysis}

Western blot bands and morphological data were quantified using ImageJ software (Image J 1.30; // https://imagej.nih.gov/ ij/) and analyzed by GraphPad Prism 5 software. Data were presented as means \pm SD. Data were analyzed by one ways ANOVA followed by post-hoc Bonferroni Multiple Comparison test using graph-pad prism-5 software. Symbols $*$ or \# or $\pi$ or $\theta$ or $\eta$ represent significant difference values $p<0.05$. Symbols $*$, \# and $\pi$ shows significant difference relative to sham. Symbol $\theta$ shows significant difference relative to I/R MCAO, $\eta$ shows significant difference between $\mathrm{p}-\mathrm{MCAO}$ and Dia $\mathrm{p}-\mathrm{MCAO}$.

\section{RESULTS}

\section{Evaluation of Neurodegeneration in Ischemic Models}

Cerebral ischemia brings robust neuronal changes in the core brain areas where dramatic blood flow reduction caused irreversible cell death. Neuronal apoptosis could be seen after $\mathrm{MCAO}$ in the ischemic penumbra or peri-infarct zone, where blood flow is less severely reduced. Sham group showed no infarction, while extensive infarction was observed for different MCAO operated rats (Figure 1B). A significant inter group variability was observed, whereas Dia p-MCAO and p-MCAO group showed extensive infarction, than I/R MCAO group relative to sham group. Further calculations of corrected \% infarcted area were higher for Dia p-MCAO (28.35 \pm 4.3$)$ than p-MCAO (22.71 $\pm 3.9 \%)$ and I/R-MCAO (11.4 \pm 3.5$)$. Unlikely no noticeable infarction was observed at hippocampus in any ischemic group (Figure 1B). The apoptotic (Caspase$3)$ and anti-apoptotic protein marker $(\mathrm{Bcl} 2)$ expression were also evaluated to validate the relative expression in different ischemic models (Figure 1C). No significant alterations were noticed in $\mathrm{I} / \mathrm{R}$ group for these markers, contrary to $\mathrm{p}$ MCAO (Figure 1C). Furthermore, Heat shock proteins (HSP) are physiological sensors that delineate extent of injury in core, penumbral and peri-infarct tissue. We therefore examined HSP70 expression by western blot. The results showed elevated expression of HSP70 in permanent and diabetic ischemic group relative to sham (Figure 1C), implies that periinfarct waves spread more sporadically in p-MCAO than $I / R$ MCAO. Moreover, western blot results were also validated by immunofluorescence findings (Figure 1D) in CA1 and CA3 region of hippocampus.

\section{NMDA Receptor Signaling}

Several reports reiterated NMDA excitotoxicity to be the fundamental cause of ischemic induced neuronal damage. Synaptic NR2a activation mediates NR2a/AKT signaling kinases, linked to CREB activation for neuronal survival (31). BDNF also demonstrated to activate several kinases including AKT (32). Our result suggested attenuated expression of NR2a and NR2b receptor subunit expression. These results further provide support to the previous observations, delineating MCAO induced calpain degradation with concurrent lower expression of neuronal survival pathways downward of NR2a (33). Moreover, calpain induced degradation and decreased 

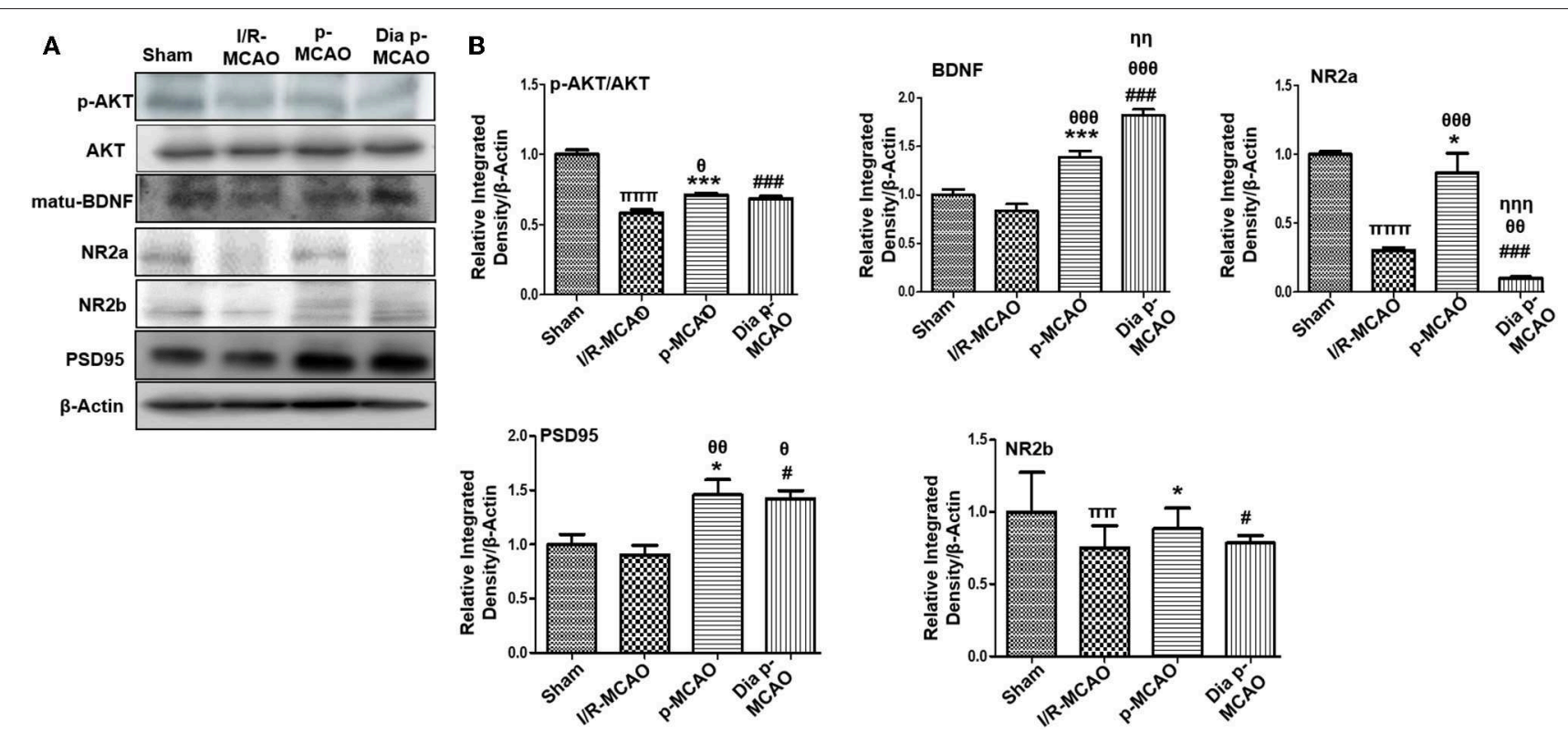

FIGURE 2 | Effect of ischemia on NMDA receptor related molecule changes (A) Representative Western blot of p-AKT, AKT, matu-BDNF, NR2a, NR2b, PSD95. (B) Histograms indicating comparative expression of various glutamate receptors in hippocampus. Densitometric analysis was expressed in relative to $\beta$-Actin $(n=$ 5/group). ${ }^{\star} p<0.05,{ }^{\#} p<0.05$, and ${ }^{\pi} p<0.05$ relative to sham group. ${ }^{\theta} p<0.05$ relative to I/R MCAO, ${ }^{n} p<0.05$ between $p-M C A O$ and Dia $p-M C A O$. ${ }^{* \star \star} p<0.001$ and \#\#\#p<0.001. ${ }^{\pi \pi}$ shows significant difference of I/R MCAO to sham, and its value is $p<0.01$, $\pi \pi \pi$ shows significant difference of I/R MCAO to sham, and its value is $p<0.001,{ }^{\theta \theta}$ shows significant difference of $\mathrm{p}-\mathrm{MCAO}$ and Dia $\mathrm{p}-\mathrm{MCAO}$ to $\mathrm{l} / \mathrm{R}$, and its value is $p<0.01$, ${ }^{\theta \theta \theta}$ shows significant difference of $\mathrm{p}-\mathrm{MCAO}$ and Dia $\mathrm{p}-\mathrm{MCAO}$ to $\mathrm{l} / \mathrm{R}$, and its value is $p<0.001$, $\eta{ }^{\prime}$ shows significant difference between $\mathrm{p}-\mathrm{MCAO}$ and Dia $\mathrm{p}-\mathrm{MCAO}$, and its value is $p<0.01$, $\eta \eta \eta$ shows significant difference between $\mathrm{p}-\mathrm{MCAO}$ and Dia $\mathrm{p}-\mathrm{MCAO}$, and its value is $p<0.001$.

expression of full length NR2a in MCAO can be reversed by increased interaction of PSD95/NR2a (34). NR2a and NR2b were differentially expressed in these ischemic models, whereas NR2a significantly downregulated in I/R and dia $\mathrm{p}$ MCAO (Figure 2), compare to p-MCAO. Unlikely, for NR2b, I/R group showed maximum cleavage than other ischemic group. The results of PSD95 is in line with NR2a expression for I/R group and p-MCAO, as PSD95 did not change in $\mathrm{I} / \mathrm{R}$, which may suggest increase susceptibility of NR2a to cleavage, while hyperexpression of PSD95 in p-MCAO may hypothetically be explained for promoting NR2a downward survival pathway.

\section{Expression of MAPK Family Protein in Ischemic Models}

Several studies demonstrated association of P38 MAPK, and c-Jun-N-terminal kinases (JNKs) to apoptotic cell death. Western blot analysis demonstrated elevated expression of activated p38 and JNK (p-JNK) in permanent and in diabetic ischemic group compared to sham and I/R operated group (Figure 3A). Furthermore, immunohistochemistry results were validated by western blot findings for $\mathrm{p}$-JNK in permanent and diabetic ischemic models (Figure 3B). Both neuroprotective and neurotoxic effects are attributed to ERK activation (35, 36). ERK is linked to neuronal injury in ischemic brain model (36). We found hyperactivated ERK in permanent and in diabetic ischemic group compared to sham operated group (Figure $\mathbf{3 A}$ ).

Moreover, Fluoro-Jade B (FJB) staining was performed to examine apoptotic cell death. Significant numbers of FJBpositive cells were noticed in $\mathrm{CA} 1, \mathrm{CA} 2$, and $\mathrm{CA} 3$ region of hippocampus in permanent and diabetic ischemic group (Figure 3C). The sham and I/R group did not exhibit significant positive staining. The FJB data is parallel to Figure 1A, where no significant changes were observed in $\mathrm{I} / \mathrm{R}$ group for Caspase- 3 and $\mathrm{Bcl} 2$.

\section{Glutamate Neurotransmission and Axonal Degeneration}

AMPA receptor activation is triggered by glutamate accumulation following cerebral ischemia. In line with previous reports, we observed significantly down expression of glutamate receptor (AMPA, GluR-1) receptor in p-MCAO groups compare to $\mathrm{I} / \mathrm{R}$ operated group in hippocampus (Figure 4). We also studied the phosphorylation of AMPA (GluR1) at serine 831 and 845. A marked increase at serine 831 were observed in p-MCAO groups (Figure 4), while no change was observed in I/R group. Moreover, serine 845 was found with aberrant expression in permanent MCAO group, and with decrease expression in diabetic and I/R (Figure 4).

Synaptosomal associated protein 25 (SNAP-25), and synaptophysin acts as synaptic marker for neuronal differentiation. To examine the detrimental effect of ischemic 


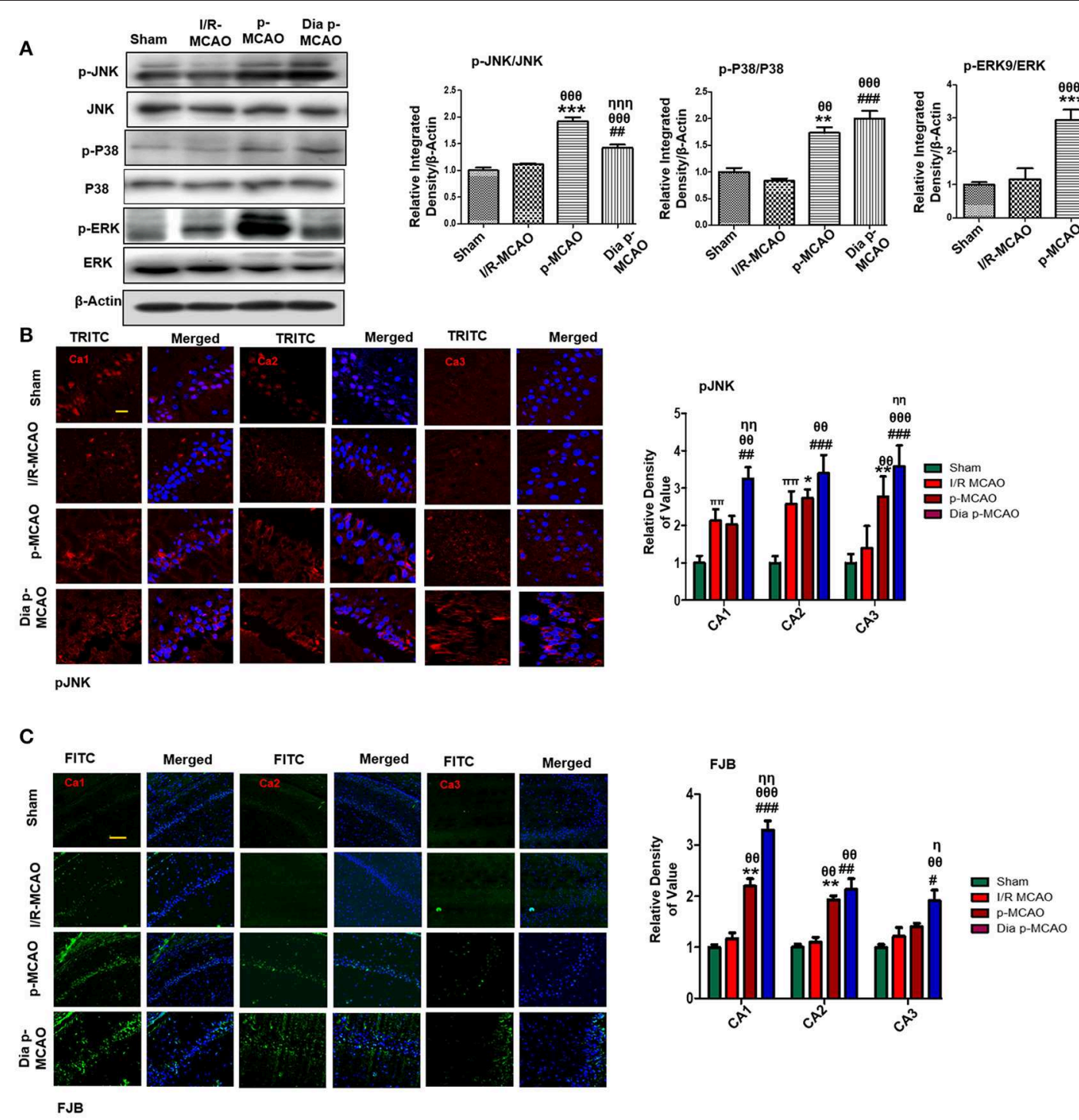

FIGURE 3 | Ischemia induced cell stress and death related signaling changes (A) Representative Western blots of p-JNK, JNK, p-P38, P38, p-ERK, ERK. Densitometric analysis was expressed in relative to $\beta$-Actin ( $n=5 /$ group). (B) Representative images of $p$-JNK immunoreactivity in CA1, CA2, and CA3 regions of hippocampus in I/R, p-MCAO and Dia p-MCAO group ( $n=5 /$ group). scale bar $=100 \mu \mathrm{m}$. (C) Representative images of FJB histochemistry showing apoptotic cells; scale bar $=30 \mu \mathrm{m}$. Significant neuronal apoptosis in CA1, CA2 and CA3 region of hippocampus was observed in $p-M C A O$ and Dia $p-M C A O$ group $(n=5 / g r o u p) . ~{ }^{\theta} p$ $<0.05$ relative to I/R MCAO, ${ }^{\eta} p<0.05$ between $\mathrm{p}-\mathrm{MCAO}$ and Dia $p-\mathrm{MCAO}$. ${ }^{*} p<0.05,{ }^{*} p<0.05$, and ${ }^{\pi} p<0.05$ relative to sham group. ${ }^{\star * \star} p<0.001$, ${ }^{* *} p<0.01$, $\# \# \# p<0.001$, and $\# \# p<0.01$. ${ }^{\theta \theta}$ shows significant difference of $\mathrm{p}-\mathrm{MCAO}$ and Dia $\mathrm{p}-\mathrm{MCAO}$ to $\mathrm{l} / \mathrm{R}$, and its value is $p<0.01$, ${ }^{\theta \theta \theta}$ shows significant difference of $\mathrm{p}-\mathrm{MCAO}$ and Dia $\mathrm{p}-\mathrm{MCAO}$ to $\mathrm{I} / \mathrm{R}$, and its value is $p<0.001,{ }^{\eta \eta}$ shows significant difference between $\mathrm{p}-\mathrm{MCAO}$ and Dia $\mathrm{p}-\mathrm{MCAO}$, and its value is $p<0.01$, ${ }^{\eta \eta \eta}$ shows significant difference between $\mathrm{p}-\mathrm{MCAO}$ and Dia $\mathrm{p}-\mathrm{MCAO}$, and its value is $p<0.001$.

damage on synaptic proteins following cerebral ischemia, we studied the expression of synaptophysin, and synaptosomalassociated protein-25 (SNAP-25) (Figure 4). It was observed that expression of SNAP-25 decreased in all ischemic operated animals (Figure 4), while no significant changes were observed in expression of synaptophysin except for dia p-MCAO (Figure 4).

\section{Effect on Inflammatory Markers}

Reports have consistently supported TLR4 activated downstream inflammatory mediators such as p-NF-кB, iNOS, and COX2 in ischemic brain injury. To evaluate whether these mediators could also be activated in hippocampus in different ischemic models, we performed western blotting. Moreover, the activation of TLR4 is linked to many downstream effects including activation of $\mathrm{p}-\mathrm{NF}-\kappa \mathrm{B}, \mathrm{iNOS}$, and COX2. Western blot results showed elevated expression of these mediators in permanent ischemic brain (Figure 5). Furthermore, the western blot data was validated by immunostaining findings for COX2 (Figure 5B). Activation of $\mathrm{p}-\mathrm{NF}-\kappa \mathrm{B}$ encodes the bulk of inflammatory mediators that exacerbate ischemic brain injury. Ischemic stroke is characterized by reactive gliosis in which 


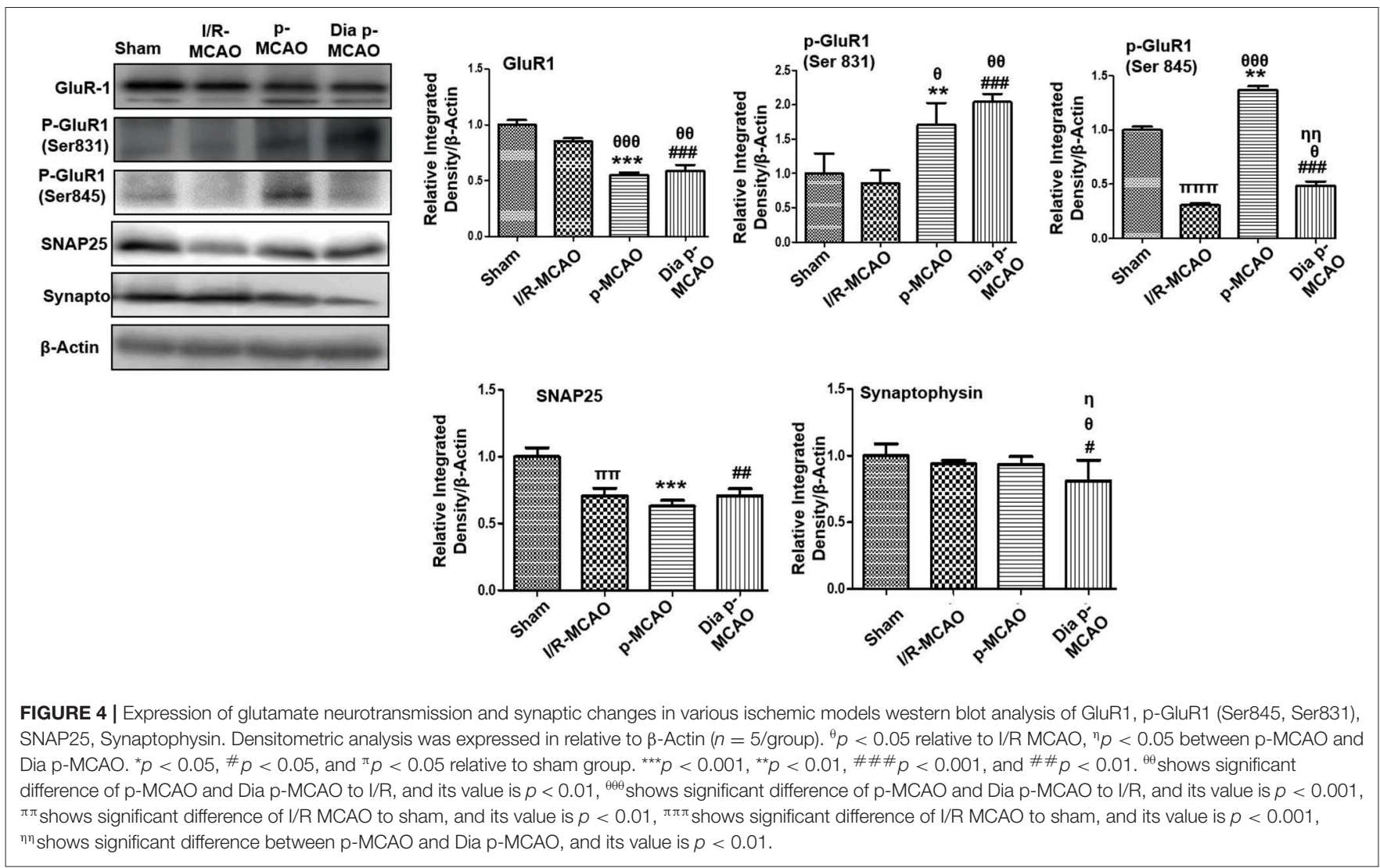

the resident microglia and astrocytes assume a characteristics cellular appearance to mediate progression of ischemic injury. These activated hypertrophic cells work as resident machinery for generating inflammatory mediators. We investigated the expression of astrocytes in ischemic hippocampus (GFAP reactive cells) following $24 \mathrm{~h}$ of permanent ischemia (Figure 5C). Immunofluorescence results revealed a significant increase in the number of GFAP reactive cells in (MCAO) compared to sham (Figure 5C).

\section{Effect on Oxidative Stress}

To further estimate oxidative stress in hippocampus, we used fluorescent dye 8-oxoguanine as oxidative stress marker. Our result demonstrated elevated expression of 8-oxoguanine particularly in diabetic ischemic group (Figure 6A). Moreover, the ubiquitously expressed nuclear erythroid 2-related factor-2 (Nrf2) is an endogenous antioxidant enzyme, translocate to nucleus for stimulating several downstream antioxidant proteins such as HO-1, superoxide dismutase (SOD) and glutathione (GSH) (37). Western blot results demonstrated elevated expression of Nrf2 and HO-1 in diabetic group (Figure 6B). VEGF is specific endothelial growth factor, implicated both in disease and normal condition. Several studies demonstrated linkage of reduced VEGF in neurodegeneration (38). Moreover, VEGF signaling, and angiogenesis are modulated by ROS formation (39). Previous studies suggested the pro-angiogenetic effect of NRF2 and down regulation of NRF2 abrogated NRF2 induced vascular sprouting (40).

To observe the expression of these growth factors in hippocampus at different ischemic intervals, we performed western blot analysis $24 \mathrm{~h}$ after MCAO (Figure 6B). Moreover, immunohistochemistry results further validate western blot findings for VEGF in permanent ischemic models (Figure 6C).

\section{DISCUSSION}

Stroke is the most devastating human health condition. Different kind of animal models are used in the laboratory to mimic human ischemic stroke with addition of different occlusion periods. Necrotic cell death primarily occurs in ischemic core, while apoptotic cell death mostly targets the penumbral tissues. The demarcation between these tissues largely depends on time of occlusion. Several studies confirmed apoptotic cell death in hippocampus after $24 \mathrm{~h}$ of ischemia in different rat strain $(15,41,42)$. Our results are parallel to previous published reports, found apoptotic cell death in hippocampus after $24 \mathrm{~h}$ of ischemia $(41,43)$. Moreover, these authors found no changes in Caspase and Bcl-2 expression in hippocampus using $\mathrm{I} / \mathrm{R}$ model. Likely, we found no changes in I/R group, but these proteins were significantly disturbed in diabetic and permanent ischemic group. 
A
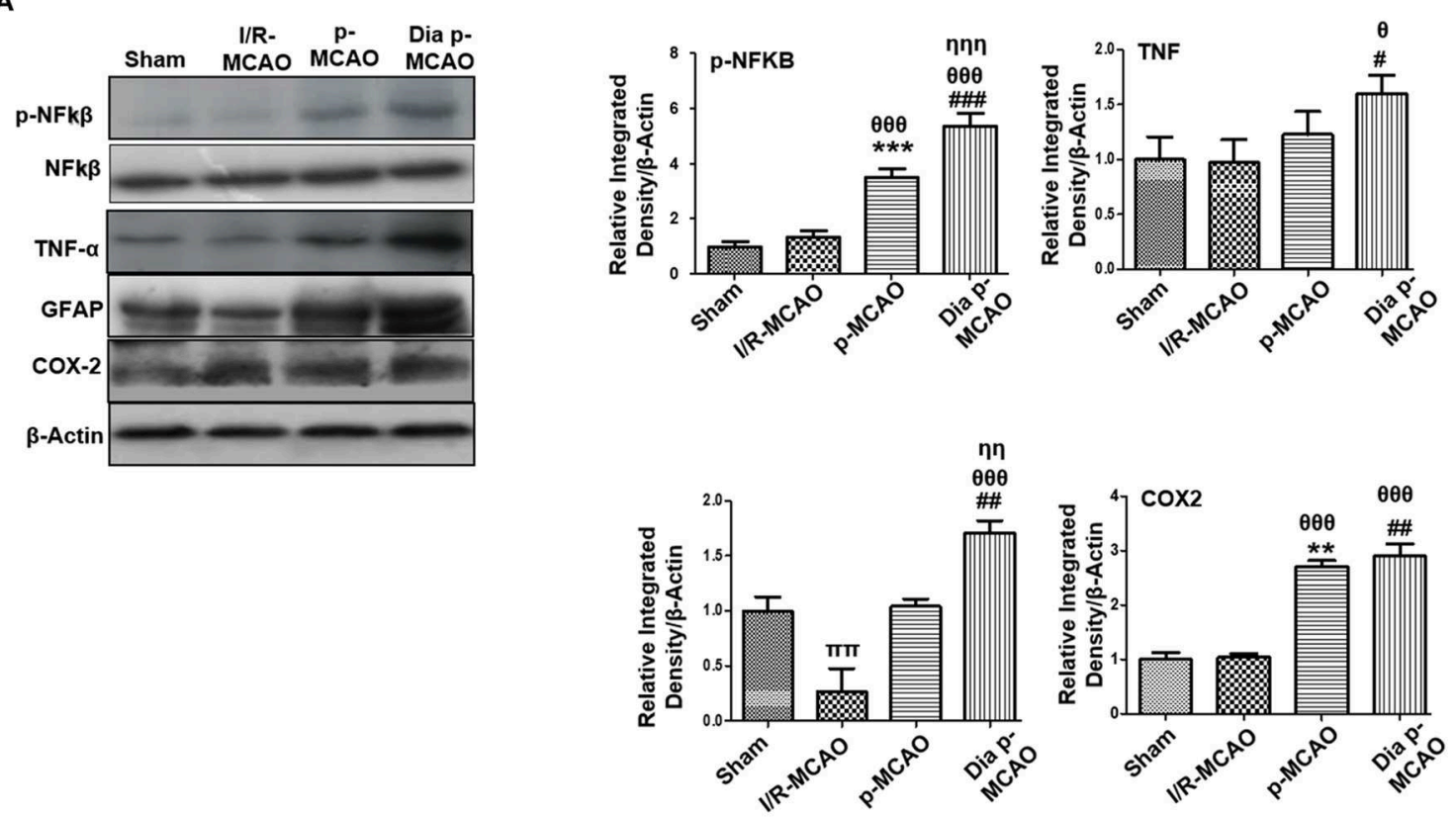

B
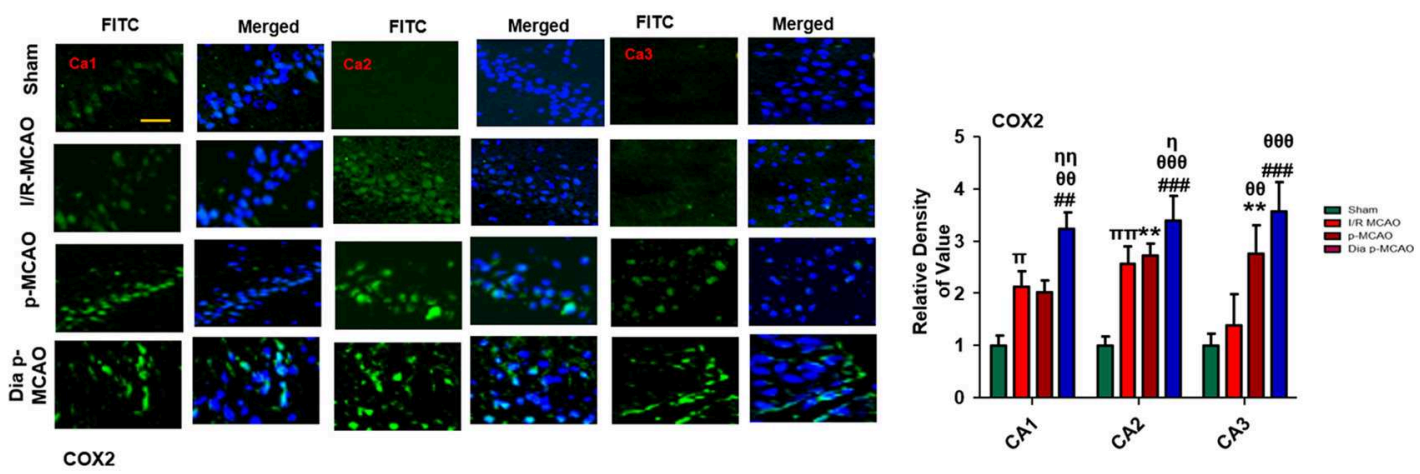

C
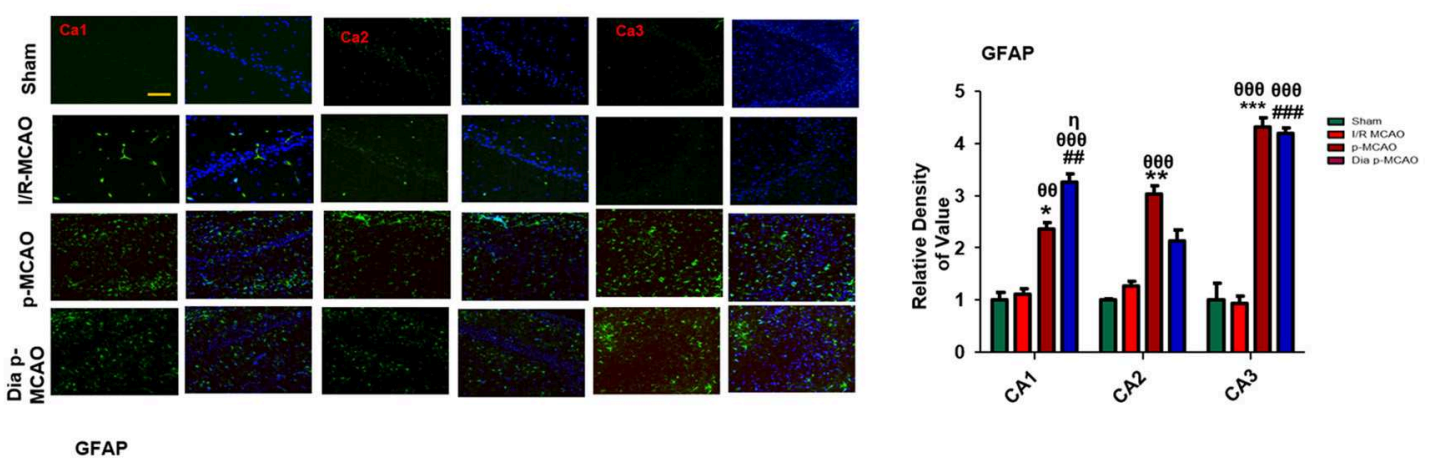

FIGURE 5 | Effect of ischemia on inflammatory related molecules in the hippocampus (A) Western blot analysis of $p-N_{k} B, N_{k} B$, TNF ${ }_{\alpha}$, GFAP, and COX2.

Densitometric analysis was expressed in relative to $\beta$-Actin $(n=5 /$ group). (B) Representative photos of immunohistochemistry for COX2, scale bar $=100 \mu \mathrm{m}$, and (C) GFAP; scale bar $=30 \mu \mathrm{m}$, ( $n=5$ /group). The CA1, CA2, and CA3 segments in p-MCAO and Dia p-MCAO showing elevated expression of COX2 and GFAP. ${ }^{\theta} p<$ 0.05 relative to I/R MCAO, ${ }^{n} p<0.05$ between $p-\mathrm{MCAO}$ and Dia $p-\mathrm{MCAO}$. ${ }^{*} p<0.05,{ }^{*} p<0.05$, and ${ }^{\pi} p<0.05$ relative to sham group. ${ }^{\star \star *} p<0.001$, ${ }^{\star *} p<0.01$, $\# \# \# p<0.001$, and \#\# $p<0.01$. ${ }^{\theta \theta}$ shows significant difference of $\mathrm{p}-\mathrm{MCAO}$ and Dia $\mathrm{p}-\mathrm{MCAO}$ to $\mathrm{l} / \mathrm{R}$, and its value is $p<0.01$, ${ }^{\theta \theta \theta}$ shows significant difference of $\mathrm{p}-\mathrm{MCAO}$ and Dia $\mathrm{p}-\mathrm{MCAO}$ to $\mathrm{I} / \mathrm{R}$, and its value is $p<0.001, \eta \eta$ shows significant difference between $\mathrm{p}-\mathrm{MCAO}$ and Dia $\mathrm{p}-\mathrm{MCAO}$, and its value is $p<0.01, \eta \eta \eta$ shows significant difference between $\mathrm{p}-\mathrm{MCAO}$ and Dia $\mathrm{p}-\mathrm{MCAO}$, and its value is $p<0.001$, ${ }^{\pi}$ shows significant difference of $\mathrm{I} / \mathrm{R} \mathrm{MCAO}$ to sham, and its value is $p<0.01$. 

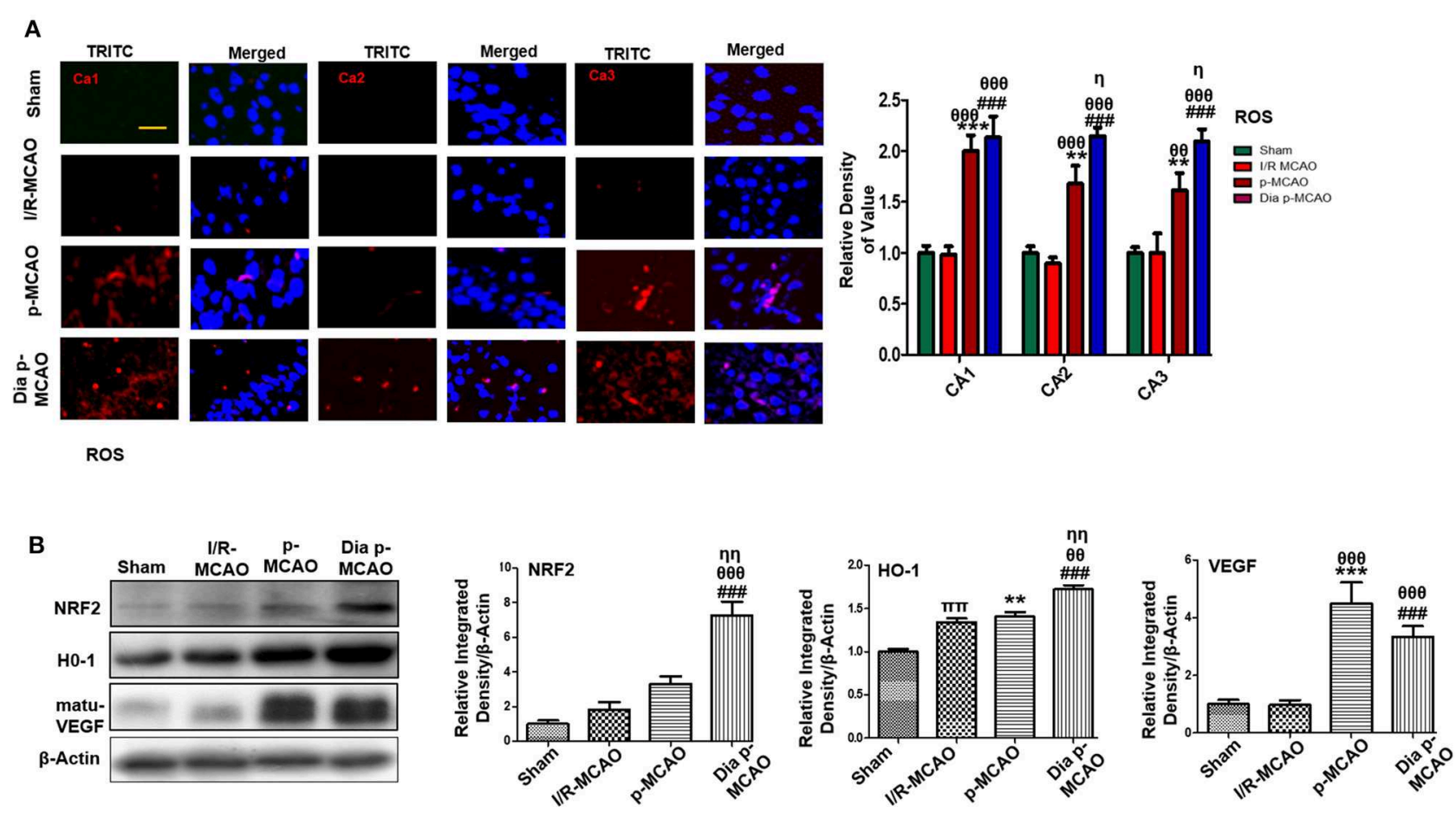

C
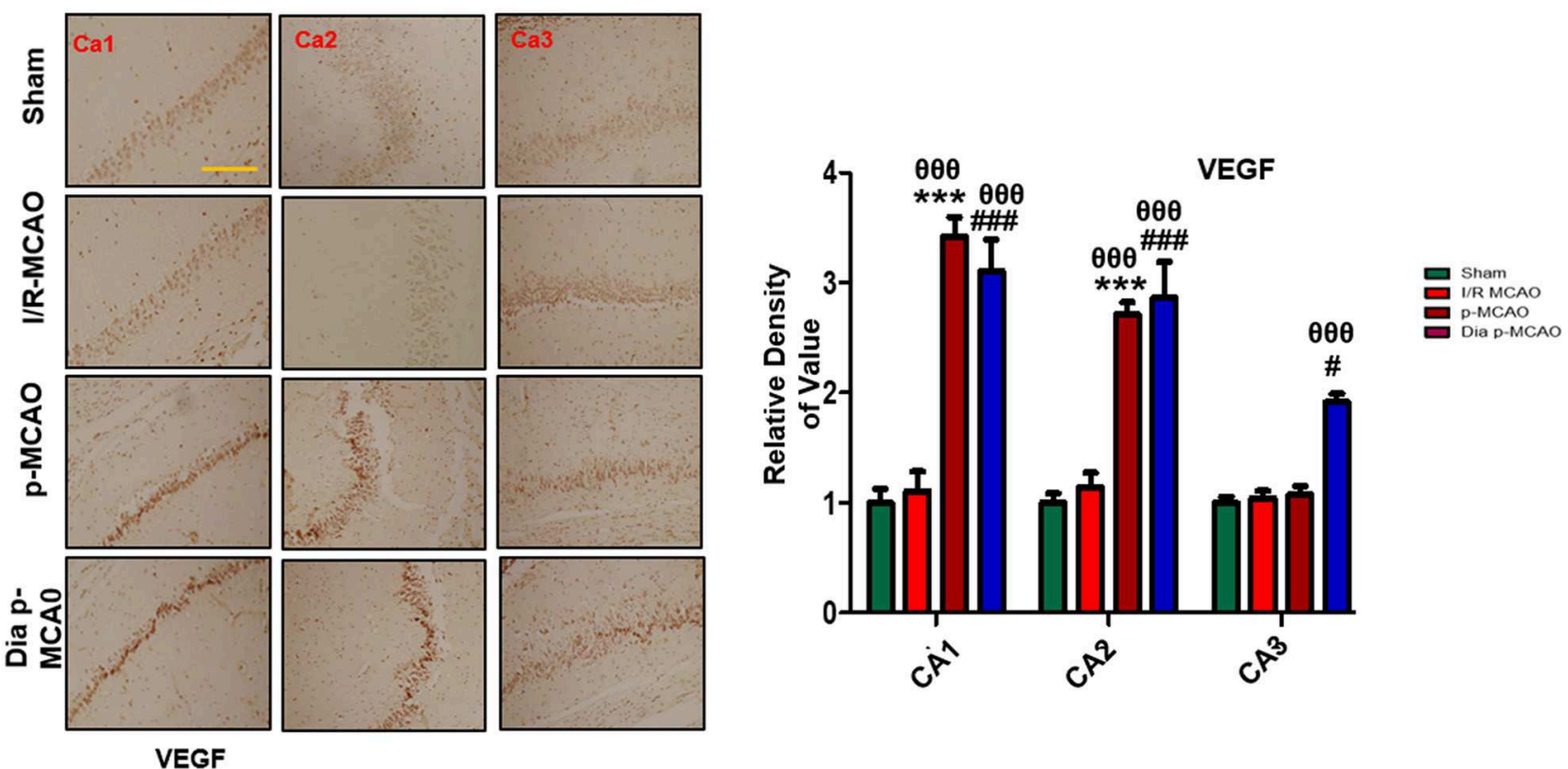

FIGURE 6 | Effect of ischemia on oxidative distress (A) Representative immunofluorescence images of 8-oxoguanine staining indicate comparative expression of ROS in various CA1, CA2, and CA3 region of hippocampus ( $n=5$ /group, Scale bar $=100 \mu \mathrm{m})$. (B) Western blot analysis of NRF2, HO-1, matu-VEGF. Densitometric analysis was expressed in relative to $\beta$-Actin ( $n=5$ /group) (C) Representative images of VEGF immunohistochemistry; scale bar $=50 \mu m$. The CA1, CA2, and CA3 segments in p-MCAO and Dia $p-M C A O$ showing elevated expression of VEGF ( $n=5 /$ group). ${ }^{\theta} p<0.05$ relative to I/R MCAO, ${ }^{n} p<0.05$ between $p-M C A O$ and Dia p-MCAO. ${ }^{\star} p<0.05,{ }^{\#} p<0.05$, and ${ }^{\pi} p<0.05$ relative to sham group. ${ }^{\star \star \star} p<0.001,{ }^{\star \star} p<0.01$, and ${ }^{\# \# \#} p<0.001$. ${ }^{\theta \theta}$ shows significant difference of $p-$ MCAO and Dia p-MCAO to $\mathrm{l} / \mathrm{R}$, and its value is $p<0.01,{ }^{\theta \theta \theta}$ shows significant difference of $\mathrm{p}-\mathrm{MCAO}$ and Dia $\mathrm{p}-\mathrm{MCAO}$ to $\mathrm{l} / \mathrm{R}$, and its value is $p<0.001, \eta \eta$ shows significant difference between $\mathrm{p}-\mathrm{MCAO}$ and Dia $\mathrm{p}-\mathrm{MCAO}$, and its value is $p<0.01$, ${ }^{\pi}$ shows significant difference of $\mathrm{I} / \mathrm{R} \mathrm{MCAO}$ to sham, and its value is $p<0.01$. 
In the current manuscript, we found significant differences in protein expression among t-MCAO, p-MCAO, and diabetic $\mathrm{p}-\mathrm{MCAO}$ at hippocampus. Our result suggested that peri-infarct localization can extend to hippocampus after $24 \mathrm{~h}$ of p-MCAO, as shown by HSP70 expression (Figures 1B,C). Hyperglycemics condition exacerbate MCAO induced brain damage by compromising vascular permeability and exacerbating neuronal toxicity. Likely, the protein expression and morphological findings in this study showed severe disturbances in diabetic and permanent ischemic group than t-MCAO. Overexpressed HSP70 indicates compromised protein synthesis due to reduced blood supply in hippocampus (44), in addition it reflects potential tissue damage (45). It is previously demonstrated that protein synthesis in penumbral and peri-infarct tissue is inhibited within hours after ischemia $(46,47)$. Induced HSP70 could also serve as endogenous protector to cope against denatured protein induced by ischemia (48).

Several reports consistently pointed out glutamate (Glu) excitotoxicity to be the fundamental hallmark of ischemic induced neurodegeneration (49). Previous results demonstrated that glutamate neurotoxicity can be observed within $2-4 \mathrm{~h}$ of ischemic occlusion $(50,51)$. In I/R MCAO models, glutamate restores to basal level quickly. However, this alteration in glutamate level can be observed for a prolonged period due to collapse of exchange pumps in p-MCAO models $(51,52)$. The role of NMDA in ischemic brain injury is not clearly known due to conflicted results $(49,53,54)$. It was suggested that NR2a subunit of NMDA lead to neuronal survival while NR2b is linked to neuronal apoptosis $(33,49)$. Furthermore, calpain is activated by ischemic injury, which degrades a large array of molecules including NMDA receptor subunits NR2a and NR2b (55). Interestingly, our results found different response patterns of NR2a and NR2b and the downstream signaling of AKT, suggesting that NMDA receptors may underlie distinct processes of glutamate transmission.

Ischemic stroke rigorously disrupts synaptic networks in hippocampus tissue. A transient ischemic occlusion is responsible for closure of about $30 \%$ synapses in hippocampus region (56). Some reports also suggested synaptic remolding after ischemic damage in hippocampus with correspondingly increased synapse formation $(57,58)$. AMPA receptor activity is controlled by several mechanisms including phosphorylation and calpain mediated cleavage of GluR1 subunit (59). In line with previous reports, we observed down expression of GluR1 in ischemic hippocampus. Phosphorylation at serine 845 is involved in the translocation of AMPA to neuronal membrane and such AMPAR phosphorylation can boost synaptic plasticity for learning and memory (60). Ischemic brain injury impairs trafficking of AMPA by down regulating (GluR1 serine 845) phosphorylation. Synaptophysin and SNAP-25 level was compromised in ischemic brain injury significantly in permanent and diabetic ischemic group similar to previous observation (61). Moreover, PSD95 showed high expression in permanent and diabetic ischemic as observed previously $(56,62)$.

Neurotropic factors are linked to neuroplasticity which accelerates neuronal repairment during ischemic brain injury. Brain derived neurotrophic factor (BDNF), vascular endothelial growth factor (VEGF) are categorized as neurotrophins having important pleiotropic effects on brain structuring. Studies suggested that BDNF and VEGF governs neurogenesis and improve functional outcomes after ischemic stroke $(63,64)$. In line with previous research, our results demonstrated hyperactive VEGF and BDNF in hippocampus. The induction of VEGF after cerebral ischemia represents inherent defense mechanism, whereby expressed VEGF leads to vascularization and sprouting of blood vessel to cope with the severe demand of energy.

Release of inflammatory proteins further exacerbate ischemic stroke injury by several mechanisms, such as activation of Toll like receptor (TLR-4) on glial cells stimulates stress kinases like (JNK and P38-MAPK) (65). Activation of these pathways triggers mitochondrial apoptotic pathway while inhibiting these kinases attenuates inflammatory cytokines (66). Cytokines are integral component of inflammation and produced largely by activated microglia, astrocytes, and neurons immediately within hour after ischemic injury. Twenty-four hours after permanent ischemia, higher expression of $\mathrm{NF}_{-\mathrm{K}} \mathrm{B}$ can trigger iNOS, COX2 production; both are toxic mediators of inflammatory cascade $(67,68)$. Besides its role in neuron inflammation, $\mathrm{NF}_{-\mathrm{K}} \mathrm{B}$ is also involved in oxidative stress via the activation of NRF2, a ubiquitous transcription factor that modulates the expression of various oxidant and antioxidant process related proteins, such as HO-1 and superoxide dismutase (SOD). Our results demonstrated elevated expression of Nrf2 and HO-1 in all three models, showing that the exacerbating effects of prolonged ischemic time and diabetic conditions.

In conclusion, our result suggests that hippocampus is adversely affected by ischemic injury after $24 \mathrm{~h}$ of intraluminal occlusion using 3/0 nylon silk. We noticed ischemic driven alteration in protein expression and histological changes in various segments of hippocampus. Furthermore, the most two commonly used MCAO models exhibited tremendous differences in terms of neuronal cell loss and neurological deficits, glutamate excitotoxic related signaling, synaptic transmission markers, neuron inflammatory and oxidative stress molecules. These differences may reflect the variations in different models, which may provide valuable information for mechanistic and therapeutic inconsistences as experienced in both preclinical models and clinical trials. More interestingly, the pathological profiles of p-MCAO in diabetic rats showed that a wide array of molecular processes are involved in the devastating effects of hyperglycaemia other than proposed changes of oxidative stress and neuron inflammation.

\section{DATA AVAILABILITY STATEMENT}

All data generated or analyzed during this study are included in this published article.

\section{ETHICS STATEMENT}

Adult male Sprague-Dawley rats weighing 200-230g (8-9 weeks) were purchased from Guangdong Medical Laboratory Animal Center, China. The experimental animals were housed at Laboratory Animal Research Center, Peking University Shenzhen 
Graduate School, under $12 \mathrm{~h}$ light $/ 12 \mathrm{~h}$ dark cycle at $18-22^{\circ} \mathrm{C}$ and had free access to diet and tap water throughout the study. The experimental procedures were set in such a way to minimize rats suffering. All experimental procedures were carried out according to the protocols approved by Institutional Animal Care and Use Committee of Peking University Shenzhen Graduate School. We did not use any blind allocation or randomization of rats, instead we adhered the criteria to keep similar weight animal to same group under the same experimental condition.

\section{AUTHOR CONTRIBUTIONS}

FS, AZ, and LK review literature and feasibility. FS, TL, GL, and XY performed surgery, western blot, and morphological

\section{REFERENCES}

1. McBride DW, Zhang JH. Precision stroke animal models: the permanent MCAO model should be the primary model, not transient MCAO. Transl Stroke Res. (2017). doi: 10.1007/s12975-017-0554-2. [Epub ahead of print].

2. Pan J, Konstas AA, Bateman B, Ortolano GA, Pile-Spellman J. Reperfusion injury following cerebral ischemia: pathophysiology, MR imaging, and potential therapies. Neuroradiology. (2007) 49:93-102. doi: 10.1007/s00234-006-0183-z

3. Sicard KM, Fisher M. Animal models of focal brain ischemia. Exp Transl Stroke Med. (2009) 1:7. doi: 10.1186/2040-7378-1-7

4. Nour M, Scalzo F, Liebeskind DS. Ischemia-reperfusion injury in stroke. Interv Neurol. (2013) 1:185-99. doi: 10.1159/000353125

5. Hossmann KA. The two pathophysiologies of focal brain ischemia: implications for translational stroke research. J Cereb Blood Flow Metab. (2012) 32:1310-6. doi: 10.1038/jcbfm.2011.186

6. Lapchak PA, Zhang JH, Noble-Haeusslein LJ. RIGOR guidelines: escalating STAIR and STEPS for effective translational research. Transl Stroke Res. (2013) 4:279-85. doi: 10.1007/s12975-012-0209-2

7. Maeda K, Hata R, Hossmann KA. Regional metabolic disturbances and cerebrovascular anatomy after permanent middle cerebral artery occlusion in C57black/6 and SV129 mice. Neurobiol Dis. (1999) 6:101-8. doi: 10.1006/nbdi.1998.0235

8. McColl BW, Carswell HV, McCulloch J, Horsburgh K. Extension of cerebral hypoperfusion and ischaemic pathology beyond MCA territory after intraluminal filament occlusion in C57Bl/6J mice. Brain Res. (2004) 997:1523. doi: 10.1016/j.brainres.2003.10.028

9. Garcia JH, Liu KF, Ho KL. Neuronal necrosis after middle cerebral artery occlusion in Wistar rats progresses at different time intervals in the caudoputamen and the cortex. Stroke. (1995) 26:636-43. doi: 10.1161/01.STR.26.4.636

10. Popp A, Jaenisch N, Witte OW, Frahm C. Identification of ischemic regions in a rat model of stroke. PLoS ONE. (2009) 4:e4764. doi: 10.1371/journal.pone.0004764

11. Nagasawa H, Kogure K. Correlation between cerebral blood flow and histologic changes in a new rat model of middle cerebral artery occlusion. Stroke. (1989) 20:1037-43. doi: 10.1161/01.STR.20.8.1037

12. Block F, Dihne M, Loos M. Inflammation in areas of remote changes following focal brain lesion. Prog Neurobiol. (2005) 75:342-65. doi: 10.1016/j.pneurobio.2005.03.004

13. Garcia JH, Liu KF, Ye ZR, Gutierrez JA. Incomplete infarct and delayed neuronal death after transient middle cerebral artery occlusion in rats. Stroke. (1997) 28:2303-10. doi: 10.1161/01.STR.28.11.2303

14. Czurko A, Nishino H. Collapsed (argyrophilic, dark) neurons in rat model of transient focal cerebral ischemia. Neurosci Lett. (1993) 12:71-4. doi: 10.1016/0304-3940(93)90562-Y

15. States BA, Honkaniemi J, Weinstein PR, Sharp FR. DNA fragmentation and HSP70 protein induction in hippocampus and cortex occurs in separate experiments. FS, AZ, LK, SK, FL, and HY performed data analysis. HY, A-UK, and PK supported the study. FS and SL designed study and wrote the manuscript. YJ and SL reviewed and approved the manuscript, and held all the responsibilities related to this manuscript. All authors reviewed and approved the manuscript.

\section{FUNDING}

This work was supported by: Grants Science and Technology Innovation Committee of Shenzhen No: JCYJ20170810163329510; Shaanxi Key Project on Science and Technology (2017SF-040); and Shandong Provincial Natural Science Foundation of China No: ZR2017MH027. neurons following permanent middle cerebral artery occlusions. J Cereb Blood Flow Metab. (1996) 16:1165-75. doi: 10.1097/00004647-199611000-00011

16. Honkaniemi J, States BA, Weinstein PR, Espinoza J, Sharp FR. Expression of zinc finger immediate early genes in rat brain after permanent middle cerebral artery occlusion. J Cereb Blood Flow Metab. (1997) 17:636-46. doi: 10.1097/00004647-199706000-00005

17. Ozdemir YG, Bolay H, Erdem E, Dalkara T. Occlusion of the MCA by an intraluminal filament may cause disturbances in the hippocampal blood flow due to anomalies of circle of Willis and filament thickness. Brain Res. (1999) 822:260-4. doi: 10.1016/S0006-8993(99)01175-0

18. Türeyen K, Vemuganti R, Sailor KA, Dempsey RJ. Ideal suture diameter is critical for consistent middle cerebral artery occlusion in mice. Neurosurgery. (2005) 56:196-200. doi: 10.1227/01.NEU.0000144490.92966.59

19. Zarow GJ, Karibe H, States BA, Graham SH, Weinstein PR. Endovascular suture occlusion of the middle cerebral artery in rats: effect of suture insertion distance on cerebral blood flow, infarct distribution and infarct volume. Neurol Res. (1997) 19:409-16. doi: 10.1080/01616412.1997.11740834

20. Bouley J, Fisher M, Henninger N. Comparison between coated vs. uncoated suture middle cerebral artery occlusion in the rat as assessed by perfusion/diffusion weighted imaging. Neurosci Lett. (2007) 412:185-90. doi: 10.1016/j.neulet.2006.11.003

21. Capes SE, Hunt D, Malmberg K, Pathak P, Gerstein HC. Stress hyperglycemia and prognosis of stroke in nondiabetic and diabetic patients: a systematic overview. Stroke. (2001) 32:2426-32. doi: 10.1161/hs1001.096194

22. Li W, Prakash R, Kelly-Cobbs AI, Ogbi S, Kozak A, El-Remessy AB, et al. Adaptive cerebral neovascularization in a model of type 2 diabetes: relevance to focal cerebral ischemia. Diabetes. (2010) 59:228-35. doi: 10.2337/db09-0902

23. Sung JH, Shah FA, Gim SA, Koh PO. Identification of proteins in hyperglycemia and stroke animal models. J Surg Res. (2016) 200:365-73. doi: $10.1016 /$ j.jss.2015.07.020

24. Shah FA, Gim SA, Kim MO, Koh PO. Proteomic identification of proteins differentially expressed in response to resveratrol treatment in middle cerebral artery occlusion stroke model. J Vet Med Sci. (2014) 76:1367-74. doi: 10.1292/jvms.14-0169

25. Shah FA, Park DJ, Gim SA, Koh PO. Curcumin treatment recovery the decrease of protein phosphatase $2 \mathrm{~A}$ subunit $\mathrm{B}$ induced by focal cerebral ischemia in Sprague-Dawley rats. Lab Anim Res. (2015) 31:134-8. doi: 10.5625/lar.2015.31.3.134

26. Shah FA, Gim SA, Sung JH, Jeon SJ, Kim MO, Koh PO. Identification of proteins regulated by curcumin in cerebral ischemia. J Surg Res. (2016) 201:141-8. doi: 10.1016/j.jss.2015.10.025

27. Shah FA, Kury LA, Li T, Zeb A, Koh PO, Liu F, et al. Polydatin attenuates neuronal loss via reducing neuroinflammation and oxidative stress in rat MCAO models. Front Pharmacol. (2019) 10:663. doi: 10.3389/fphar.2019.00663

28. Shah FA, Liu G, Al Kury LT, Zeb A, Abbas M, Li T, et al. Melatonin protects MCAO-induced neuronal loss via NR2A mediated prosurvival pathways. Front Pharmacol. (2019) 10:297. doi: 10.3389/fphar.2019.00702 
29. Al Kury LT, Zeb A, Abidin ZU, Irshad N, Malik I, Alvi AM, et al. Neuroprotective effects of melatonin and celecoxib against ethanol-induced neurodegeneration: a computational and pharmacological approach. Drug Des Devel Ther. (2019) 13:2715-27. doi: 10.2147/DDDT.S207310

30. Shah FA, Zeb A, Ali T, Muhammad T, Faheem M, Alam SI, et al. Identification of proteins differentially expressed in the striatum by melatonin in a middle cerebral artery occlusion rat model-a proteomic and in silico approach. Front Neurosci. (2018) 12:888. doi: 10.3389/fnins.2018.00888

31. Terasaki Y, Sasaki T, Yagita Y, Okazaki S, Sugiyama Y, Oyama N, et al. Activation of NR2A receptors induces ischemic tolerance through CREB signaling. J Cereb Blood Flow Metab. (2010) 30:1441-9. doi: $10.1038 /$ jcbfm. 2010.18

32. Hetman M, Kanning K, Cavanaugh JE, Xia Z. Neuroprotection by brainderived neurotrophic factor is mediated by extracellular signal-regulated kinase and phosphatidylinositol 3-kinase. J Biol Chem. (1999) 274:22569-80. doi: $10.1074 /$ jbc. 274.32 .22569

33. Chen M, Lu TJ, Chen XJ, Zhou Y, Chen Q, Feng XY, et al. Differential roles of NMDA receptor subtypes in ischemic neuronal cell death and ischemic tolerance. Stroke. (2008) 39:3042-8. doi: 10.1161/STROKEAHA.108.521898

34. Gascon S, Sobrado M, Roda JM, Rodriguez-Pena A, Diaz-Guerra M. Excitotoxicity and focal cerebral ischemia induce truncation of the NR2A and NR2B subunits of the NMDA receptor and cleavage of the scaffolding protein PSD-95. Mol Psychiatry. (2008) 13:99-114. doi: 10.1038/sj.mp.4002017

35. Xia Z, Dickens M, Raingeaud J, Davis RJ, Greenberg ME. Opposing effects of ERK and JNK-p38 MAP kinases on apoptosis. Science. (1995) 270:1326-31. doi: $10.1126 /$ science. 270.5240 .1326

36. Alessandrini A, Namura S, Moskowitz MA, Bonventre JV. MEK1 protein kinase inhibition protects against damage resulting from focal cerebral ischemia. Proc Natl Acad Sci USA. (1999) 96:12866-9. doi: 10.1073/pnas.96.22.12866

37. Jung KA, Kwak MK. The Nrf2 system as a potential target for the development of indirect antioxidants. Molecules. (2010) 15:7266-91. doi: $10.3390 /$ molecules 15107266

38. Storkebaum E, Carmeliet P. VEGF: a critical player in neurodegeneration. $J$ Clin Invest. (2004) 113:14-8. doi: 10.1172/JCI20682

39. Chua CC, Hamdy RC, Chua BH. Upregulation of vascular endothelial growth factor by $\mathrm{H}_{2} \mathrm{O}_{2}$ in rat heart endothelial cells. Free Radic Biol Med. (1998) 25:891-7. doi: 10.1016/S0891-5849(98)00115-4

40. Wei Y, Gong J, Thimmulappa RK, Kosmider B, Biswal S, Duh EJ. Nrf2 acts cell-autonomously in endothelium to regulate tip cell formation and vascular branching. Proc Natl Acad Sci USA. (2013) 110:E3910-8. doi: 10.1073/pnas.1309276110

41. Chung JY, Yi JW, Kim SM, Lim YJ, Chung JH, Jo DJ. Changes in gene expression in the rat hippocampus after focal cerebral ischemia. J Korean Neurosurg Soc. (2011) 50:173-8. doi: 10.3340/jkns.2011.50.3.173

42. Genovese T, Mazzon E, Paterniti I, Esposito E, Bramanti P, Cuzzocrea S. Modulation of NADPH oxidase activation in cerebral ischemia/reperfusion injury in rats. Brain Res. (2011) 1372:92-102. doi: 10.1016/j.brainres.2010.11.088

43. Butler TL, Kassed CA, Sanberg PR, Willing AE, Pennypacker KR. Neurodegeneration in the rat hippocampus and striatum after middle cerebral artery occlusion. Brain Res. (2002) 929:252-60. doi: 10.1016/S0006-8993(01)03371-6

44. Mies G, Ishimaru S, Xie Y, Seo K, Hossmann KA. Ischemic thresholds of cerebral protein synthesis and energy state following middle cerebral artery occlusion in rat. J Cereb Blood Flow Metab. (1991) 11:753-61. doi: 10.1038/jcbfm.1991.132

45. Basu S, Binder RJ, Suto R, Anderson KM, Srivastava PK. Necrotic but not apoptotic cell death releases heat shock proteins, which deliver a partial maturation signal to dendritic cells and activate the NF-kappa B pathway. Int Immunol. (2000) 12:1539-46. doi: 10.1093/intimm/12.11.1539

46. Zhang F, Liu CL, Hu BR. Irreversible aggregation of protein synthesis machinery after focal brain ischemia. J Neurochem. (2006) 98:102-12. doi: 10.1111/j.1471-4159.2006.03838.x

47. Schubring-Giese M, Leemburg S, Luft AR, Hosp JA. Protein synthesis inhibition in the peri-infarct cortex slows motor recovery in rats. PLoS ONE. (2016) 11:e0157859. doi: 10.1371/journal.pone.0157859
48. Weinstein PR, Hong S, Sharp FR. Molecular identification of the ischemic penumbra. Stroke. (2004) 35(11 Suppl. 1):2666-70. doi: 10.1161/01.STR.0000144052.10644.ed

49. Liu Y, Wong TP, Aarts M, Rooyakkers A, Liu L, Lai TW, et al. NMDA receptor subunits have differential roles in mediating excitotoxic neuronal death both in vitro and in vivo. J Neurosci. (2007) 27:2846-57. doi: 10.1523/JNEUROSCI.0116-07.2007

50. Matsumoto K, Lo EH, Pierce AR, Halpern EF, Newcomb R. Secondary elevation of extracellular neurotransmitter amino acids in the reperfusion phase following focal cerebral ischemia. J Cereb Blood Flow Metab. (1996) 16:114-24. doi: 10.1097/00004647-199601000-00014

51. Taguchi J, Graf R, Rosner G, Heiss WD. Prolonged transient ischemia results in impaired $\mathrm{CBF}$ recovery and secondary glutamate accumulation in cats. J Cereb Blood Flow Metab. (1996) 16:271-9. doi: 10.1097/00004647-199603000-00012

52. Kogure K, Yamasaki Y, Matsuo Y, Kato H, Onodera H. Inflammation of the brain after ischemia. Acta Neurochir Suppl. (1996) 66:40-3. doi: 10.1007/978-3-7091-9465-2_7

53. Margaill I, Parmentier S, Callebert J, Allix M, Boulu RG, Plotkine M. Short therapeutic window for MK-801 in transient focal cerebral ischemia in normotensive rats. J Cereb Blood Flow Metab. (1996) 16:107-13. doi: 10.1097/00004647-199601000-00013

54. Gutierrez-Vargas JA, Munoz-Manco JI, Garcia-Segura LM, CardonaGomez GP. GluN2B N-methyl-D-aspartic acid receptor subunit mediates atorvastatin-Induced neuroprotection after focal cerebral ischemia. J Neurosci Res. (2014) 92:1529-48. doi: 10.1002/jnr.23426

55. Simpkins KL, Guttmann RP, Dong Y, Chen Z, Sokol S, Neumar RW, et al. Selective activation induced cleavage of the NR2B subunit by calpain. $J$ Neurosci. (2003) 23:11322-31. doi: 10.1523/JNEUROSCI.23-36-11322.2003

56. Kovalenko T, Osadchenko I, Nikonenko A, Lushnikova I, Voronin K, Nikonenko I, et al. Ischemia-induced modifications in hippocampal CA1 stratum radiatum excitatory synapses. Hippocampus. (2006) 16:814-25. doi: 10.1002/hipo.20211

57. Hammond C, Crepel V, Gozlan H, Ben-Ari Y. Anoxic LTP sheds light on the multiple facets of NMDA receptors. Trends Neurosci. (1994) 17:497-503. doi: 10.1016/0166-2236(94)90140-6

58. Jourdain P, Nikonenko I, Alberi S, Muller D. Remodeling of hippocampal synaptic networks by a brief anoxia-hypoglycemia. J Neurosci. (2002) 22:310816. doi: 10.1523/JNEUROSCI.22-08-03108.2002

59. Yuen EY, Liu W, Yan Z. The phosphorylation state of GluR1 subunits determines the susceptibility of AMPA receptors to calpain cleavage. J Biol Chem. (2007) 282:16434-40. doi: 10.1074/jbc.M701283200

60. Esteban JA, Shi SH, Wilson C, Nuriya M, Huganir RL, Malinow R. PKA phosphorylation of AMPA receptor subunits controls synaptic trafficking underlying plasticity. Nat Neurosci. (2003) 6:136-43. doi: 10.1038/nn997

61. Ishimaru H, Casamenti F, Ueda K, Maruyama Y, Pepeu G. Changes in presynaptic proteins, SNAP-25 and synaptophysin, in the hippocampal CA1 area in ischemic gerbils. Brain Res. (2001) 903:94-101. doi: 10.1016/S0006-8993(01)02439-8

62. Martone ME, Jones YZ, Young SJ, Ellisman MH, Zivin JA, Hu BR. Modification of postsynaptic densities after transient cerebral ischemia: a quantitative and three-dimensional ultrastructural study. J Neurosci. (1999) 19:1988-97. doi: 10.1523/JNEUROSCI.19-06-01988.1999

63. Sun Y, Jin K, Xie L, Childs J, Mao XO, Logvinova A, et al. VEGFinduced neuroprotection, neurogenesis, and angiogenesis after focal cerebral ischemia. J Clin Invest. (2003) 111:1843-51. doi: 10.1172/JCI200 317977

64. Greenberg ME, Xu B, Lu B, Hempstead BL. New insights in the biology of BDNF synthesis and release: implications in CNS function. J Neurosci. (2009) 29:12764-7. doi: 10.1523/JNEUROSCI.3566-09.2009

65. Kacimi R, Giffard RG, Yenari MA. Endotoxin-activated microglia injure brain derived endothelial cells via NF-kappaB, JAK-STAT and JNK stress kinase pathways. J Inflamm. (2011) 8:7. doi: 10.1186/1476-9 255-8-7

66. Munoz L, Ralay Ranaivo H, Roy SM, Hu W, Craft JM, McNamara LK, et al. A novel p38 alpha MAPK inhibitor suppresses brain proinflammatory cytokine up-regulation and attenuates synaptic dysfunction and behavioral deficits 
in an Alzheimer's disease mouse model. J Neuroinflammation. (2007) 4:21. doi: 10.1186/1742-2094-4-21

67. Caso JR, Pradillo JM, Hurtado O, Lorenzo P, Moro MA, Lizasoain I. Toll-like receptor 4 is involved in brain damage and inflammation after experimental stroke. Circulation. (2007) 115:1599-608. doi: 10.1161/CIRCULATIONAHA.106.603431

68. Yao L, Kan EM, Lu J, Hao A, Dheen ST, Kaur C, et al. Toll-like receptor 4 mediates microglial activation and production of inflammatory mediators in neonatal rat brain following hypoxia: role of TLR4 in hypoxic microglia. J Neuroinflammation. (2013) 10:23. doi: 10.1186/1742-209 4-10-23
Conflict of Interest: The authors declare that the research was conducted in the absence of any commercial or financial relationships that could be construed as a potential conflict of interest.

Copyright $\odot 2019$ Shah, Li, Kury, Zeb, Khatoon, Liu, Yang, Liu, Yao, Khan, Koh, Jiang and Li. This is an open-access article distributed under the terms of the Creative Commons Attribution License (CC BY). The use, distribution or reproduction in other forums is permitted, provided the original author(s) and the copyright owner(s) are credited and that the original publication in this journal is cited, in accordance with accepted academic practice. No use, distribution or reproduction is permitted which does not comply with these terms. 